\title{
LA RENTA BÁSICA INCONDICIONAL ANTE LAS LIMITACIONES DE LAS RENTAS MÍNIMAS
}

\section{Why a basic income can succeed where minimum income schemes fall short}

Julen Bollain URbieta*1, Daniel Raventós Pañella ${ }^{2}$

1 Instituto Hegoa, Universidad del País Vasco / Euskal Herriko Unibertsitatea

${ }^{2}$ Departamento de Sociología, Facultat d'Economia i Empresa, Universitat de Barcelona

\section{RESUMEN}

En el presente artículo realizamos, a partir de los parcelados estudios existentes, una revisión integral y detallada acerca de la eficacia y las limitaciones de los programas de rentas mínimas. Asimismo, procedemos a analizar cuál es el comportamiento de la renta básica incondicional ante las deficiencias estructurales detectadas en los programas de rentas mínimas.

Palabras clave: rentas mínimas, garantía de ingresos, renta básica incondicional, renta básica, subsidios condicionados, libertad, políticas sociales.

\section{ABSTRACT}

In this article we carry out, taking as a start point the existing divided studies, an extensive comprehensive review of the effectiveness and shortcomings of minimum income programs. Likewise, we analyze how an unconditional basic income would behave when facing the structural deficiencies previously detected in such minimum income programs.

Keywords: minimum income, guaranteed income, unconditional basic income, basic income, means-tested benefits, freedom, social policies.

* Correspondencia a: Julen Bollain Urbieta. Instituto Hegoa. Avda. Lehendakari Agirre, 81. 48015 Bilbao-Bizkaia - bollainjulen@ gmail.com / jbollain001@ikasle.ehu.eus - https://orcid.org/0000-0002-1298-5666

Cómo citar: Bollain Urbieta, Julen; Raventós Pañella, Daniel. (2018). «La renta básica incondicional ante las limitaciones de las rentas mínimas»; Lan Harremanak, 40, 90-114. (https://doi.org/10.1387/lan-harremanak.20332).

Recibido: 4 noviembre, 2018; aceptado: 10 diciembre, 2018.

ISSN 1575-7048 - elSSN 2444-5819 / @ 2018 UPV/EHU

(c) (i) Esta obra está bajo una licencia

Creative Commons Atribución 4.0 Internacional 


\section{SUMARIO}

1. Introducción.-2. Rentas mínimas o de garantía de ingresos.-3. El debate sobre la eficacia de las rentas mínimas.-4. Limitaciones de las rentas mínimas. a) Restricciones presupuestarias. b) Errores de cobertura. c) Estigmatización de las personas beneficiarias. d) Costes de administración. e) Trampa de la pobreza.-5. La Renta Básica Incondicional-Una reforma radical. a) Pequeña historia de la Renta Básica Incondicional: del siglo XVI al siglo xIx. b) El auge de la renta básica incondicional: siglo xx. c) La globalización e incorporación de la renta básica incondicional en la agenda política: siglo xxi.-6. Comparación renta básica incondicional y rentas mínimas.-7. Conclusiones.-8. Bibliografía.

\section{Introducción}

Durante las últimas décadas los programas de rentas mínimas focalizados en sujetos de derecho en situaciones de necesidad han proliferado a lo largo y ancho del continente Europeo. Los programas de rentas mínimas son, básicamente, prestaciones económicas asistenciales y condicionadas a cierta situación de necesidad que funcionan como última red del Estado de Bienestar. Es decir, una vez las personas han «fracasado» o han "caído», las rentas mínimas, de manera más o menos extraordinaria, actuarán ex post ofreciendo unos ingresos a cambio, habitualmente, de una deuda social por las prestaciones percibidas.

Según Nelson (2010), al menos dos procesos han contribuido a este incremento. El primero se basa en los cambios en la distribución de los ingresos, que han tenido como resultado que la ciudadanía dependa cada vez más de las rentas mínimas para asegurar su subsistencia. El segundo proceso consiste en el cambio gradual de las prioridades políticas. Los responsables políticos han utilizado la focalización de las rentas mínimas como mecanismo para redefinir las responsabilidades sociales en la actualidad.

En contraposición a las rentas mínimas, nos encontramos con la renta básica incondicional. Una asignación monetaria pública y regular, otorgada de forma incondicional a toda la población. De esta manera, su fin es garantizar la existencia material de toda la ciudadanía por el mero hecho de existir y como derecho de ciudadanía. 
$\mathrm{Al}$ tiempo en el que se acrecienta el debate sobre los errores estructurales que los programas de rentas mínimas contienen, son cada vez más voces que, tanto desde el mundo académico o del político, como desde el empresarial o del activista, abogan por la implantación de una renta básica incondicional.

Con el fin de ofrecer un análisis comparativo entre los programas de rentas mínimas y la renta básica incondicional frente a los problemas estructurales de los primeros, hemos dividido el artículo en tres grandes bloques. Primero realizaremos una recopilación histórica sobre los programas de rentas mínimas, analizaremos su eficacia y examinaremos sus limitaciones (restricciones presupuestarias, errores de cobertura, estigmatización de las personas beneficiarias, costes de administración y trampa de la pobreza). En el segundo bloque ahondaremos en la definición y en la historia de la renta básica incondicional desde sus orígenes hasta la actualidad, período en el que de forma vertiginosa se ha establecido en la agenda política y en el que, tal y como afirma Van Parijs en el documental RBUI, nuestro derecho a vivir (Orús, 2018), en un solo año (2017) se ha hablado más sobre la renta básica incondicional que en toda la historia de la humanidad. Finalmente, concluiremos con un análisis sobre el comportamiento de la renta básica incondicional ante los errores previamente detectados en los programas de rentas mínimas.

\section{Rentas mínimas o de garantía de ingresos}

Los programas de rentas mínimas o garantía de ingresos son prestaciones económicas asistenciales cuya finalidad es que toda persona cuente con lo mínimo para poder vivir dignamente. Al ser prestaciones asistenciales, todas ellas comparten la característica de ser otorgadas al sujeto de derecho siempre y cuando éste haya probado una insuficiencia de ingresos o de activos que le permita tener acceso a dicho programa de rentas mínimas o de garantía de ingresos (Cohen y Friedman, 1972). Es decir, son prestaciones económicas condicionadas a cierta situación de necesidad establecida ex-ante (means-tested), y cuya lógica no es otra que ofrecer una ayuda ex post a las personas que se encuentren en dicha situación de necesidad y puedan demostrar a la Administración Pública que son «merecedoras» de tutela ex post.

Además, tal y como constata Euzeby (1991) en "Le revenu minumum garanti», para beneficiarse de los programas de rentas mínimas o de garantía de ingresos, además de la forzosa situación de necesidad del sujeto de derecho, también se requieren requisitos adicionales que condicionan aún más el acceso a éstos. Entre estos requisitos, habitualmente pueden encontrarse los relativos a la edad del sujeto de derecho, a su residencia o a la nacionalidad o a ciertas condiciones de participación en medidas de formación, orientación e intermediación 
laboral para "garantizar que la incorporación al mercado de trabajo sea una opción más atractiva y rentable que la simple percepción de prestaciones económicas de garantía de ingresos» (Ley 18/2008).

De esta manera, podemos afirmar que la finalidad de los programas de rentas mínimas o de garantía de ingresos es proporcionar unos medios temporales para cubrir una carencia de ingresos, presumiblemente transitoria, y estando vinculados, en gran medida, a un proceso de intervención social. Además, dicho proceso de intervención social se encuentra estrechamente ligado a actividades de inserción laboral porque, mientras las rentas mínimas ostentan un carácter extraordinario, lo ordinario es lograr la subsistencia a través de un (o más de un) trabajo asalariado.

Pero, ¿cuál es el origen de estos programas? En primer lugar cabe mencionar que, a finales de la Segunda Guerra Mundial, los programas de asistencia social se consideraban una parte agonizante de los Estados de Bienestar europeos ya que, la inclusión social se entendía debía conseguirse mediante el pleno empleo. Debido a este pensamiento y a las favorables condiciones socioeconómicas, durante las siguientes décadas se dio un considerable aumento en la cobertura de los sistemas de seguridad social en la mayoría de los países europeos, abarcando así a una mayor parte de la población (Therborn, 1995; Crouch, 1999).

Esta lógica del pleno empleo y expansión de los sistemas de seguridad social fue cumpliéndose a lo largo de los ańos 50 y 60, reduciendo enormemente la necesidad de sistemas de asistencia social (en aquellos países donde ya estaban establecidos). Sin embargo, quedaban aún países de Europa del Sur donde estos sistemas no se habían implantado (Aust y Arriba, 2005).

Cuando en los años 70 las tasas de desempleo empezaron a incrementar y se convirtieron en un grave problema para la ciudadanía de los países (Blanchard y Wolfers, 2000), los sistemas de asistencia social volvieron a aparecer en la agenda política y social, así como el debate sobre la necesidad de incrementar su cobertura dentro de los Estados de Bienestar.

Es en este nuevo marco cuando en 1988 se aprueba en Francia la Ley que materializa el Revenu Minimum d'Insertion (RMI), un sistema de garantía de ingresos que busca blindar el «derecho a obtener un medio de existencia adecuado» para «cualquier persona que, debido a su edad, su estado fisico o mental, el estado de la economía y el empleo» no pueda trabajar remuneradamente (Loi n. ${ }^{\circ 88-1088) . ~}$

El RMI es, sin duda, un punto de inflexión que se presenta como alternativa a los sistemas de asistencia social basados exclusivamente en una prestación económica de las décadas de la posguerra. En este nuevo escenario de altas tasas de desempleo, el RMI establece los dos pilares que impregnarán los sistemas de garantía de ingresos que pronto se propagarán por los estados del sur de Europa (Espańa, Portugal o Italia). Por un lado, la dimensión monetaria y, por 
otro lado, la dimensión de la inclusión laboral. Asimismo, al observar las reformas y los cambios llevados a cabo en la mayoría de los estados europeos (Suecia, Holanda, Noruega, Dinamarca, Alemania, Finlandia o Reino Unido) en años posteriores, se puede apreciar cómo las políticas de "activación» obligatorias también se fortalecieron (Aust y Arriba, 2005), ligando la garantía de ingresos a acciones de inclusión laboral y entendiendo dichos procesos como parte esencial hacia la restauración del vínculo social de las personas beneficiarias. De este modo, y tal y como aseveraron Lødemel y Trickey (2001), la asistencia social atraviesa la barrera de ser un derecho social para convertirse en «una oferta que no podrás rechazar». Además, en este contexto la tradicional separación entre políticas pasivas y activas de empleo empieza a difuminarse.

\section{El debate sobre la eficacia de las rentas mínimas}

El objetivo principal de las rentas mínimas desde su creación ha sido, y es, el de eliminar o reducir la pobreza (Immervoll, 2010). Sin embargo, y debido al no estrictamente alto grado de cumplimiento de dicho objetivo, el debate académico sobre la efectividad de las rentas mínimas es copioso.

Sainsbury y Morissens (2002) publicaron un estudio sobre la efectividad de los subsidios condicionados en Europa a mediados de la década de los 90, cuando las tasas de pobreza incrementaron considerablemente (pasando la barrera de los 50 millones de personas por debajo del umbral de la pobreza en Europa según datos de EUROSTAT (1994)) y éstos tomaron un papel central a la hora de tratar de reducir la misma. Dicho estudio demostraba que los subsidios condicionados per se fueron insuficientes para conseguir una gran reducción de la pobreza a mediados de los 90 en Europa, donde la reducción de la tasa de la pobreza por países osciló entre el $0,2 \%$ en Italia y el 8,5\% en Reino Unido. Adicionalmente al Reino Unido, los países donde los subsidios condicionados redujeron en un mayor porcentaje la pobreza fueron Suecia (5,5\%), Finlandia $(5,1 \%)$ y República Checa $(4,2 \%)$. En el otro extremo se encuentran Hungría $(1,8 \%)$, Francia $(1,7 \%)$ y Bélgica $(0,8 \%)$. Por el contrario, dicho estudio evidenció cómo las transferencias sociales sí tuvieron, en general, un rol mucho más significativo que los subsidios condicionados a la hora de reducir la pobreza en la Europa de mediados de los 90 (comparación de la tasa de pobreza antes y después de impuestos).

Trabajos posteriores, como el realizado por Bahle, Pfeifer y Wendt (2010), constatan que en el año 2006, aún muchos países europeos mantenían las cuantías de sus rentas mínimas por debajo del umbral de la pobreza, lo que imposibilita que las familias que reciben estas prestaciones superen la misma. El grado de suficiencia de las cuantías de las prestaciones, medido a través de la tasa de ade- 
cuación, determina el nivel en que la prestación proporciona a las personas recursos suficientes para garantizar un nivel de vida adecuado y permita llevar una vida que sea compatible con la dignidad humana (Frazer y Marlier, 2016). Las mayores tasas de adecuación de dichas rentas mínimas (incrementada con las ayudas para la vivienda) corresponden a Reino Unido, Irlanda, Alemania, Dinamarca, Países Bajos, Finlandia y Suecia. Además de estos países, algunos otros de Europa Oriental también cuentan con rentas mínimas superiores al umbral de la pobreza nacional (Hungría, Polonia y República Checa). Es, sin embargo, en España y Portugal donde éstas son menos generosas, contando con rentas mínimas por debajo del umbral de la pobreza nacional.

Además de en la tasa de adecuación de los sistemas de las rentas mínimas, éstos también difieren en los efectos de reducción de la pobreza que conllevan al conjunto de la ciudadanía. En la literatura existente se encuentran suficientes indicios que demuestran cómo el gasto social que realiza un Estado mantiene una correlación negativa con la tasa de pobreza en dicha área geográfica. Sin embargo, y aunque el impacto que el Estado de Bienestar realiza sobre los niveles de desigualdad y la tasa de la pobreza es muy significativo, no sucede lo mismo con el impacto de la asistencia social, el cual es mucho más modesto (Korpi y Palme, 1998).

Korpi y Palme (1998) llamaron "paradoja de la distribución» al hecho de que cuanto más focalizados estén en las personas en situación de pobreza los subsidios condicionados, existe una probabilidad menor para reducir la pobreza y la desigualdad (the more targeted at the poor, the less likely to reduce poverty and inequality). Es decir, cuanto más dirigidos estén los subsidios condicionados a situaciones de pobreza, menor será la redistribución que se realice hacia las familias de rentas bajas.

Como hemos podido constatar, el debate sobre si la eficacia en la redistribución de la riqueza es mayor a través de los subsidios condicionados o a través de los subsidios incondicionales es muy recurrente. Gran parte de la literatura existente se centra en los resultados distributivos de las rentas mínimas y se ha planteado casi exclusivamente en términos de cómo se distribuye el gasto público presupuestado. Así, los debates se han centrado generalmente en estudiar la forma en la que se asigna el gasto público presupuestado, dejando de lado, por ejemplo, la importancia de la cuantía del dinero disponible para su distribución.

En este sentido, un análisis exhaustivo de la literatura académica actual pasaría por examinar aquellas razones que provocan que las rentas mínimas no funcionen adecuadamente. En este artículo identificamos cinco principales limitaciones que analizaremos detalladamente a continuación: las restricciones presupuestarias, la cobertura insuficiente, la estigmatización de las personas beneficiarias, los costes de administración y la trampa de la pobreza. 


\section{Limitaciones de las rentas mínimas}

\section{a) Restricciones presupuestarias}

Es innegable que uno de los principales problemas de los subsidios condicionados, especialmente de los programas de rentas mínimas, es la limitación y la inestabilidad presupuestaria que se les adjudica.

En general, con estos programas se cubre un porcentaje muy pequeño de la población debido a que se presupuesta una cantidad escasa teniendo en cuenta la totalidad de personas que pudieran ser potenciales beneficiarias. Además de esto, hay que tener en cuenta que el presupuesto que se va a destinar a dichos programas no es fijo, por lo que las diferentes estructuras institucionales podrán reducir o incrementar el presupuesto que se destine a los mismos. Un caso especialmente notorio es el que ha ocurrido en los países europeos durante la última década. Desde que la crisis tuviera inicio en 2008, las presiones para reducir el déficit presupuestario de los países miembros han sido significativas, lo que a su vez, ha reducido mayoritariamente el presupuesto para las prestaciones dirigidas a las personas en situaciones de pobreza (Ministerio de Hacienda, 2018).

Autores como Korpi y Palme (1998) apuntan que sin tener en cuenta la forma funcional o las diversas variables que también pudieran tener relevancia, la fórmula redistributiva que indica el grado de redistribución es una función multiplicativa entre el grado de condicionalidad a las rentas bajas y la cuantía total presupuestada para redistribuir. Por lo tanto, podemos afirmar que la existencia de presupuestos restrictivos en materia de subsidios condicionados minorará la reducción de las tasas de pobreza que éstos realicen a través de la redistribución de la riqueza. En consonancia con lo que afirmaba Van Oorschot (1991), parte de la ineficacia redistributiva de las rentas mínimas, formuladas sobre premisas socialdemócratas, reside en el momento en que exista una tensión entre los objetivos de las mismas y la implementación de dichos objetivos (es decir, cuando ésta se base en concepciones liberales y de contención del gasto público).

\section{b) Errores de cobertura}

Mientras que los errores de cobertura que conllevan los subsidios condicionados pueden tomar la forma de un "pago en exceso" del subsidio a personas que no cumplen los requisitos para acceder a ellos (y que así se habría demostrado si hubieran presentado toda la información sobre sus rentas y otras características relevantes), lo que estudiaremos más exhaustivamente a continuación son los errores de cobertura respecto a las personas con derecho a acceder a dichos subsidios.

Actualmente los subsidios condicionados no ofrecen una cobertura completa de la totalidad de la ciudadanía que cumple los requisitos necesarios para 
acceder a los mismos. Esto es lo que denominaremos tasa de no-aceptación ${ }^{1}$ (non take-up rate), definida como la tasa de las personas que no perciben los subsidios condicionados a los cuales tienen derecho (Matsaganis et al., 2010).

Pese a que en Europa el interés por el estudio de la tasa de no-aceptación de los subsidios condicionados ha incrementado en la última década, diversos autores (Van Oorschot, 1991; Atkinson, 1996 o Riphahn, 2000) han apuntalado que éste es uno de los temas más desatendidos en la literatura académica. Una posible explicación a esta falta de interés es que a diferencia del "pago en exceso", que es costoso para las finanzas de la Administración Pública, la tasa de no-aceptación ahorra dinero público (Matsaganis et al., 2010).

Van Oorschot (1998) sostenía que una de las reflexiones que las distintas percepciones de las sociedades occidentales comparten en relación con el problema de la no-aceptación es, precisamente, que éste no es un problema de primer nivel. La idea central es dar por hecho que muy poca gente de la población que cumpla los requisitos para acceder a dicho derecho no hará uso de él, ya que las personas buscarán la ganancia y no rechazarán deliberadamente la prestación. Según esta percepción, los pocos casos de no-aceptación que pudieran existir tendrían su origen o bien en un cálculo realizado deliberadamente por las personas con acceso a dicho derecho y en base al cual no creen que les convenga hacer el esfuerzo que el subsidio condicionado conlleva, o bien en el hecho de no querer depender de aquello que consideran caridad.

Los porcentajes de la tasa de no-aceptación existentes en la literatura actual varían en función del país y en función de la fecha en la que se ha realizado el estudio. En Alemania, la tasa de no-aceptación del programa de asistencia social llamado Sozialhilfe se situó, entre las personas, en el 58,7\% en 1991 y en el 52,3\% en 1995 (Neumann y Hertz, 1998). Esta tasa se vería incrementada hasta el 63,1\% en los núcleos familiares en el año 1993 (Riphanh, 2000) y al 59,6\% en 1996 (Bird et al., 1999). En Francia, entre los años 1994 y 1996, la tasa de no-aceptación de los programas de rentas mínimas Revenu Minimum d'Insertion y Allocation de Parent Isolé se encontró entre el 35\% y el 48\% (Terracol, 2002). Van Oorschot (1995) analizó el programa de renta mínima de Holanda, Bijzondere Bijstand, obteniendo como resultado que la tasa de no-aceptación se estableció, en el año 1990, entre el 53\% y el 63\%. El estudio realizado por Bargain, Immervoll y Viitamäkki (2012) que analizó la tasa de no-aceptación del relativamente generoso programa de rentas mínimas finés, Toimeentulotuki, tuvo como resultado que ésta se encontró entre el $40 \%$ y el $50 \%$ durante los años 1996 y 2003. En Escocia se analizó el programa de rentas mínimas llamado Income Support, sobre el cual se estimó que en 1996 contaba con una tasa de no-aceptación entre el 30\% y el 50\% (Bramley et al., 2000).

1 Traducción propia. 
Pero es Suecia el país de Europa donde la medición de la tasa de no-aceptación ha obtenido mayor porcentaje. El estudio realizado por Gustafsson (2002) revela que en el año 1985 la tasa de no-aceptación del programa de asistencia social de este país se encontraba en el 82,2\% y en 1997 ésta descendió hasta el 77,3\%.

Los resultados mencionados arriba coinciden con el informe realizado a raíz de estudios anteriores (tanto académicos como administrativos) por Hernanz, Malherbet y Pellizzari (2004), donde podemos apreciar cómo la tasa de no-aceptación de las prestaciones sociales, en general, suele ser muy alta en los países de la OCDE. En especial, ésta es mayor cuando hace referencia a los programas de asistencia social condicionada, entre los que se encuentran las rentas mínimas y cuyo porcentaje varía entre el $20 \%$ y el $60 \%$. Es decir, podemos afirmar que pese a que la tasa de no-aceptación de los programas de rentas mínimas varía considerablemente dependiendo del país (ya que algunos programas están mejor diseñados que otros), ésta siempre es realmente significativa.

El fracaso de las sucesivas campañas publicitarias en torno a los subsidios condicionados muestra, además, que la alta tasa de no-aceptación de los mismos no se debe simplemente a información inadecuada, sino que las razones de que existan personas en situaciones que les permitirían el acceso a dichos programas y no lo hagan, son múltiples. Es un hecho que en las sociedades actuales nos encontramos con numerosas objeciones a reclamar subsidios condicionados.

\section{c) Estigmatización de las personas beneficiarias}

De manera recurrente se asegura en la literatura que una de las razones principales por la que la tasa de acceso a los subsidios condicionados se reduce considerablemente es la estigmatización asociada con los mismos (véase, por ejemplo, Standing, 2003 o Stuber y Kronebusch, 2004). Asimismo, tal y como afirmaba Nelson (2007), el hecho de asociar la estigmatización con los subsidios condicionados y la reducción de la tasa de aceptación de los mismos contiene, a su vez, consecuencias negativas para la mitigación de la pobreza.

Muchas veces se habla de un estigma social para describir la situación en la que se encuentran las personas que acceden a un programa de rentas mínimas. Dicha situación fue explicada por Rainwater en 1982, cuando argumentó que a los ojos de gran parte de la sociedad, ser pobre constituye una forma de desviación social. Y ello es así porque en el imaginario colectivo se ha asentado la creencia de que salir de la pobreza no solo es posible, sino que además es digno de elogio. Como consecuencia de esta creencia, las personas que reciben asistencia pública han sido etiquetadas, a lo largo de los ańos, como vagas, carentes de ambición, perezosas y moralmente débiles. «Perdedoras», en definitiva.

Parte de estas percepciones negativas existentes hacia las personas beneficiarias de asistencia social han sido fomentadas, parcialmente, a través de los me- 
dios de comunicación (Gilens, 1999). Un estudio realizado en Estados Unidos que refleja de forma meridiana la evolución de cómo se ha retratado desde los medios de comunicación a las personas en situación de pobreza, rastreó la representación mediática que se hizo de las mismas tanto en prensa escrita como en noticiarios de televisión desde 1950. Durante el inicio de la segunda mitad del siglo XX, entre 1950 y 1964, el retrato de la pobreza era predominantemente a través de hombres blancos. Sin embargo, entre 1967 y 1992, las personas negras representaban de media el $57 \%$ de las personas en situación de pobreza representada en los medios. Una cifra que casi doblaba el porcentaje de las personas negras que verdaderamente se encontraban en situación de pobreza en Estados Unidos. Pero este porcentaje se elevó a su máximo exponente en 1972 y en 1973, años en los que la opinión pública hacia el estado de bienestar era mayormente negativa y en los que entre el $70 \%$ y el $75 \%$ de las personas que aparecían haciendo referencia a situaciones de pobreza eran afroamericanas. En este sentido, el estudio concluye que la tergiversación que se materializa a través de los medios de comunicación, contribuye de manera significativa a manipular la opinión de la ciudadanía en cuanto a los programas dirigidos a personas en situación de pobreza.

Jacobs y Saphiro (1994) demostraron que existe un amplio consenso en la sociedad estadounidense a favor de que el gobierno garantice a toda la ciudadanía un seguro médico ya que la atención médica es «una necesidad fundamental de la vida humana que se debe a todos los estadounidenses». Así, programas como Medicaid pueden ser percibidos como menos susceptibles al abuso por parte de las personas beneficiarias ya que éstas simplemente reciben beneficios de atención médica que se presume están limitados a ciertas necesidades definidas, en lugar de beneficios en efectivo que podrían gastarse de "forma inapropiada" (Cook y Barrett, 1992).

La percepción de abuso en la sociedad contribuye a una estereotipación de las personas que reciban cualquier prestación. Pero el grado de estigmatización también difiere en cuanto a la naturaleza de los programas.

La focalización de los subsidios suele ser socialmente divisiva en la medida en que divide a la sociedad en aquellas personas que dan y en aquéllas que reciben (Currie, 2004), lo que genera un estigma social hacia aquellas personas que (potencialmente) puedan recibir. Riphahn (2000) citó cuatro variables mediante las cuales se puede aproximar el grado de estigmatización que una unidad familiar puede sufrir y que, sin duda, también afectan a la tasa de no-aceptación. Las dos primeras son variables que se ciñen a la composición de la unidad familiar y las dos últimas son variables externas a la misma.

En primer lugar encontramos la edad y el sexo de la persona considerada cabeza de familia. A esta variable definida por Riphahn, habría que añadir el efecto que podría tener sobre ella la construcción social de los mandatos de gé- 
nero de dicha sociedad. Habitualmente, en las sociedades occidentales, las normas sociales establecen un mayor estigma en aquellos hombres (en comparación con las mujeres) que no pueden proporcionar recursos suficientes a su familia.

La segunda variable que afecta a la unidad familiar es la existencia o no de hijos e hijas. La motivación de padres y madres a la hora de otorgar unos medios de subsistencia suficientes a los niños y a las niñas tiene una correlación positiva con la tasa de aceptación de las rentas mínimas.

La tercera variable a la hora de aproximar el grado de estigmatización sería el tamaño de la comunidad en la que se resida. En las comunidades pequeñas ( $<20.000$ habitantes) es más dificultoso proteger la información sobre la dependencia económica que la unidad familiar pueda tener respecto a la Administración Pública. Así, mientras se espera que en las comunidades pequeñas la tasa de no-aceptación pueda incrementar por la posible estigmatización, en las ciudades grandes se aplica el efecto opuesto. Es decir, en las ciudades grandes se espera que el anonimato pueda proteger a las personas beneficiarias de la estigmatización.

Finalmente, existe la connotación negativa que se pueda tener en ciertas sociedades respecto la asistencia social. En lugares donde la connotación tiene tintes negativos, el nivel de estigma que las personas beneficiaras llevan consigo es mayor y por tanto, la tasa de no-aceptación, también será mayor.

Además, habría que añadir a estas cuatro variables explicitadas por Riphahn (2000) que los sistemas de rentas mínimas son, a través de los mecanismos de control, uno de los más claros ejemplos de invasión en las vidas de las personas por parte de la Administración Pública. Este control continuo (desde el momento en que se solicita la prestación hasta el momento en que se deja de recibirla) e invasivo contribuye a una mayor estigmatización de las personas (potencialmente) beneficiarias, quienes tienen que «desnudarse» y actuar como «sumisas suplicantes» de una renta mínima ante la burocracia estatal.

En definitiva, y en línea con lo expuesto por Stuber y Kronebusch (2004), observamos dos formas de estigmatización: de integridad y de tratamiento. La estigmatización de integridad se basa en las preocupaciones sobre la posibilidad de estereotipación negativa (personas vagas, perezosas, deshonestas...) que se asocia a los subsidios condicionados y que afecta a la imagen que la persona (potencialmente) beneficiaria tiene sobre sí misma. La estigmatización de tratamiento, por el contrario, se basa en cómo las demás personas ven y actúan ante las personas beneficiarias que, una vez estigmatizadas, son etiquetadas como responsables de su destino (Weiner $e t$ al., 1988). Y tanto el temor a ser estereotipado y estigmatizado, como el temor a ser tratado de manera hostil mientras dure la participación en programas de rentas mínimas, tienen una correlación positiva con la tasa de no-aceptación. 


\section{d) Costes de administración}

El hecho de dirigir las prestaciones a los sectores más vulnerables de la sociedad, es decir, el hecho de que las prestaciones sean condicionadas, hace que de facto existan costes administrativos significativos (Benfield, 2007). Dichos costes administrativos conllevan una reducción en la cuantía de los recursos destinados a paliar la pobreza. Los programas de subsidios condicionados, como son los programas de rentas mínimas, para su correcta materialización y posterior seguimiento requieren tanto de personal y habilidades como de tiempo y dinero.

En la actualidad, en la era de la transferencia tecnológica y con una administración razonablemente bien gestionada, la mayor parte del coste administrativo asociado de los programas de rentas mínimas va dirigido al coste de la información y al coste del control (Van Parijs, 2006). Es decir, aquél destinado a informar a la ciudadanía de sus derechos y a verificar que las personas (potencialmente) beneficiarias de dichos derechos realmente son elegibles para acceder a los mismos. Además, un programa de subsidios condicionados va a requerir un control periódico de las rentas o activos que pueda tener una unidad familiar beneficiaria para comprobar su legitimidad de acceso al programa, por lo que estos costes aumentarán cuanto mayor sea el control que la administración realice a lo largo del proceso.

En términos económicos, gran parte de la ineficiencia de los subsidios condicionados es debida a los costes de administración (Atkinson, 1996) como muestra de una atroz racionalización de las políticas sociales (Raventós, 2006) y de los recursos públicos de toda la ciudadanía.

Autores como Son (2008), que abogan por la introducción de programas de subsidios condicionados en escenarios con presupuestos limitados, afirman que en ocasiones, lo que consideran beneficios inherentes a la condicionalidad pueden verse superados por los costes administrativos necesarios para llevar a cabo el proceso de focalización de las prestaciones a un determinado grupo de la ciudadanía y el control de las acciones que las personas beneficiarias realizan. A medida que se incrementan los condicionantes para una focalización más exacta hacia el grupo en cuestión (con el objetivo de reducir los "pagos en exceso" y la tasa de no-aceptación), también aumentan los costes administrativos.

En la literatura actual no existen multitud de estudios empíricos acerca del porcentaje de los costes administrativos en los programas de subsidios condicionados debido a la gran dificultad y a las imprecisiones a la hora de calcularlos. Sin embargo, sí podemos evidenciar que la media de los costes administrativos en programas de subsidios condicionados en América Latina es de un 9\% (Grosh, 1994), lo que se asemeja bastante a los diversos estudios realizados a nivel global (véase Benfiel, 2007; Devereux et al., 2017 o Van der Berg et al., 2010). Es decir que, de media, si cogiéramos un presupuesto hipotético de 
$100 €$, 9€ irían directamente dirigidos a sufragar gastos administrativos en vez de a paliar la pobreza, reduciendo así la capacidad redistributiva de los subsidios condicionados.

\section{e) Trampa de la pobreza}

En general, las rentas mínimas son unas prestaciones condicionadas que no son acumulativas. Son prestaciones que, como mucho, complementan una posible renta que la unidad familiar pueda tener hasta un umbral fijado por el programa en cuestión (que, lógicamente, varía dependiendo del programa/país). Esta no acumulabilidad se materializa en una deducción de la cuantía de la prestación en consonancia con la cuantía de ingresos de los que pueda disponer la unidad familiar (Bollain, 2017).

La imposibilidad de recibir al mismo tiempo dos rentas (por incompatibilidad expresa del programa de rentas mínimas en cuestión), nos deriva a la trampa de la pobreza. La trampa de la pobreza es definida como «la penalización que comporta el aceptar por parte del beneficiario de un subsidio condicionado un trabajo remunerado» (Raventós, 2000), siendo dicha penalización la cuantía reducida de la prestación condicionada.

Pongamos un ejemplo. Noelia es una mujer que percibe un subsidio condicionado de manera mensual tras haber agotado todas las prestaciones contributivas a las que tenía derecho. A Noelia le ofrecen un empleo con una remuneración mensual y deberá analizar si le resulta conveniente o no su aceptación ya que a cambio de aceptar el empleo ofrecido, Noelia verá reducida la cuantía del subsidio condicionado o incluso podrá perderlo completamente.

Supongamos que la cuantía que percibe Noelia proviene de una renta mínima y asciende a $650 € /$ mes. El empleo que le ofrecen, a jornada completa, tiene como remuneración mensual $900 €$ netos, una cuantía cercana al salario más frecuente en el Estado español para el año 2018 y que reciben unas 500.000 personas asalariadas. Ante esta situación, como decíamos en el párrafo anterior, Noelia deberá analizar la conveniencia de aceptar este empleo o no, porque ella solo aceptará un trabajo remunerado cuando se materialice en unos ingresos que permitan superar la anteriormente citada trampa de la pobreza. Es decir, cuando el trabajo remunerado le aporte unos ingresos netos superiores a los que pierde.

Si aceptase el empleo ofrecido en este supuesto, la renta mínima de 650€/ mes le sería reducida de manera drástica ya que, como mucho, ésta complementaría los $900 €$ mensuales provenientes del empleo hasta el umbral que fijara el programa. Es decir, tal y como sostiene Raventós (2007), se perdería parte de cada unidad monetaria de prestación por cada unidad monetaria de ingreso salarial que pudiera percibirse a cambio de la aceptación del empleo. Pero dado 
que habitualmente, en el Estado español, este umbral que fijan los programas de rentas mínimas no suele elevarse hasta los $900 €$, la renta mínima le sería arrebatada completamente por la Administración Pública competente ya que no cumpliría los requisitos para acceder a la misma.

Más allá del preciso análisis coste-beneficio monetario, habría que añadir otras variables adicionales que también influirían de manera significativa en la ecuación para la aceptación del empleo o no. Entre otros factores encontramos el esfuerzo que hay que realizar para desarrollar todo trabajo remunerado, la autoestima que puede ofrecer el desarrollo de un trabajo remunerado y el estigma social que puede acarrear el no desarrollarlo o las características del trabajo en cuestión. Además habría que añadir al análisis las actividades alternativas que debería sacrificar Noelia por aceptar dicho trabajo remunerado (ya sean actividades de ocio, de trabajos domésticos y de cuidados o de trabajo voluntario) o el ingreso neto extra que recibiría por hora trabajada. Es decir, el ingreso por hora trabajada en el empleo en relación a la renta mínima que percibe en la actualidad. Si aceptase el empleo de $900 € /$ mes, ingresaría $250 €$ más en comparación a la cuantía de $650 € /$ mes que ingresa a través de la renta mínima en la actualidad. Siendo el empleo de jornada completa, suponemos que Noelia trabajaría de manera remunerada 160 horas mensuales (40 semanales) por un ingreso neto extra de $250 € / \mathrm{mes}$. O lo que es lo mismo, en comparación con la situación actual, Noelia recibiría $1,56 €$ de ingreso neto extra por cada hora trabajada en el empleo que le han ofertado.

Este supuesto no dista mucho de la realidad. Analicemos dos casos reales que se dan en la actualidad en Cataluña y en Navarra. Una familia en Cataluña, al encontrarse todos sus miembros en edad de trabajar en situación de desempleo, percibe a través de la Renta Garantizada de Ciudadanía 1.200€/mes. Si a uno de ellos se le ofrece un empleo de $900 € /$ mes, no encontraría incentivo alguno para aceptarlo ya que esto implicaría la suspensión inmediata de la Renta Garantizada de Ciudadanía y tendría que esperar 6 meses para poder volver a percibirla. En Navarra, sin embargo, si una persona en situación de desempleo cobrase $600 € /$ mes de la Renta Garantizada de Navarra y encontrase un trabajo a tiempo parcial de $400 € /$ mes, pasaría a cobrar $520 € /$ mes de la renta garantizada. Por lo que en total ingresaría $920 € /$ mes. Si al cabo de un tiempo le ofrecieran pasar a jornada completa y cobrar $800 € /$ mes, la renta garantizada se reduciría a $160 € /$ mes y pasaría a cobrar $960 € /$ mes. Es decir, 40 euros netos extra al mes por trabajar 20 horas más a la semana. O lo que es lo mismo, $0,5 €$ de ingreso neto extra por cada hora trabajada a jornada parcial (Arcarons et al, 2018).

Tal y como exponen Raventós (2007) y Arcarons, Raventós y Torrens (2018), la trampa de la pobreza concebida a raíz de la condicionalidad en los subsidios ha de considerarse un desincentivo o estímulo negativo a la hora de buscar la activación de las personas beneficiarias y que éstas acepten ofertas de 
trabajo remunerado de baja cuantía (en este caso, una cuantía cercana al salario más frecuente del Estado español) o a tiempo parcial.

Pero mientras la no acumulabilidad en los subsidios desincentiva la búsqueda activa de empleo, incentiva el pequeño fraude fiscal. Adicionalmente, muchas veces los beneficios a corto plazo de obtener dos rentas, una a través de la prestación y otra a través de una actividad realizada en el ámbito del trabajo sumergido, tienen una mayor influencia que los prejuicios a medio y largo plazo, donde el trabajo sumergido jamás se tendrá en cuenta a la hora de calcular una prestación de desempleo o una pensión de jubilación (Raventós, 2007).

\section{La Renta Básica Incondicional-Una reforma radical}

En contraposición a los programas de rentas mínimas o de garantía de ingresos como herramientas que proporcionen unos medios temporales para cubrir una carencia de ingresos presumiblemente transitoria, la alternativa que más interés y debate ha suscitado entre los distintos autores y autoras ha sido la renta básica incondicional (Van Parijs, 2006). La renta básica incondicional es definida como un «ingreso pagado por el estado, como derecho de ciudadanía, a cada miembro de pleno derecho o residente de la sociedad incluso si no quiere trabajar de forma remunerada, sin tomar en consideración si es rico o pobre [...]. En menos palabras: una renta básica es una asignación monetaria pública incondicional a toda la población»(Red Renta Básica, 2018).

\section{a) Pequeña historia de la Renta Básica Incondicional: del siglo XVI al siglo XIX}

A tenor de la definición, la renta básica incondicional es una idea simple y sencilla. Sin embargo, ésta cuenta con un background diverso y su principal fin, históricamente, no ha sido el de erradicar la pobreza. El concepto de renta básica incondicional, explicado con otros términos y formas pero con el mismo trasfondo, nos remonta hasta la llegada del renacimiento, época de humanistas como el español Juan Luís Vives y su libro De Subventione Pauperum. Sive de humanis necessitatibus libri II (1526) o el inglés Tomás Moro, quien en Utopia (Moro, 1516) argumentaba que para acabar con los robos lo más útil era otorgar a toda la ciudadanía medios de subsistencia para que no tuvieran que convertirse en ladrones y posteriormente, como castigo, tener que colgarlos en la plaza del pueblo.

Sin embargo, es durante los dos últimos siglos cuando distintos economistas, sociólogos, filósofos o políticos han ido sofisticando y estudiando detalladamente la renta básica y se ha conseguido introducir en la agenda social y política ya en pleno siglo xx. A finales del siglo XVIII y a principios del siglo XIX, el debate teórico sobre la renta básica se basaba en la naturaleza «igualitaria» del con- 
cepto, el cual era descrito por sus defensores como una forma de recompensa justa por la apropiación de bienes comunes por parte de una minoría privilegiada. Así, al calor de la revolución francesa es cuando Thomas Paine (1797) discutió en Justicia Agraria los orígenes de la propiedad y el hecho de que la tierra es una propiedad común de toda la humanidad. Paine declaró que una vez la tierra es cultivada es el valor añadido lo que se convierte en propiedad individual y no la tierra en sí, ya que ésta no tenía propietario alguno originariamente. A raíz de esta afirmación el inglés escribió que «cada propietario, por tanto, de terrenos cultivados adeuda a la comunidad una renta del suelo por el terreno que ocupa). Paine abogaba por un pago a cada persona al alcanzar la edad de 21 años como compensación parcial por la "pérdida de su herencia natural causada por la introducción del sistema de propiedad territoriali, más un pago regular anualmente a cada persona que llegase a los 50 años de edad. Medio siglo después y siguiendo un razonamiento parecido, el socialista belga Joseph Charlier (1848) publicó el libro Solution au problème social ou constitution humanitaire, basée sur la loi naturelle, et précédée de l'exposé de motifs, donde aseguraba que toda persona tenía derecho a un «dividendo territorial» que correspondiese al valor per cápita del territorio nacional y sus recursos naturales. Autores como Van Parijs (2006) consideran el «dividendo territorial» de Charlier como la primera formulación de una renta básica pura y genuina pese a que la alegación de Charlier apenas fue escuchada y él mismo fue olvidado velozmente.

\section{b) El auge de la renta básica incondicional: siglo $\mathrm{xx}$}

Será ya en el siglo xx cuando empiecen a darse debates sobre la renta básica incondicional particularmente en Inglaterra y en Estados Unidos (Van Parijs y Vanderborght, 2017).

En el escenario de entreguerras en Inglaterra, Dennis Milner y su mujer Mabel Milner (1918) publicaron el folleto Scheme for a State Bonus y en julio de ese mismo año formaron la State Bonus League para impulsar la idea de un dividendo estatal (State Bonus) dentro del partido laborista. Aupado por la State Bonus League, Dennis Milner consiguió elevar el debate del dividendo estatal al Congreso Nacional del Partido Laborista en 1920 y finalmente la medida sería rechazada por el partido en 1921. Pocos años después, un círculo de intelectuales cercanos al partido laborista con George D. H. Cole a la cabeza, trataron de difundir el debate en torno a lo que primeramente denominaron dividendo social (social dividend) y más adelante renta básica (basic income). Este debate se ciñó a Inglaterra y pronto pasaría al olvido hasta los años 60 sin mayores resultados tangibles.

En la década de 1960, el debate sobre propuestas parecidas a la renta básica incondicional llegaría a la política estadounidense a través de una petición impulsada en 1968 por más de cien economistas estadounidenses (entre ellos Ja- 
mes Tobin, John Kenneth Galbraith o Paul Samuelson) y que dio lugar al Plan de Asistencia Familiar (Family Assistance Plan) bajo la administración Nixon. La propuesta consistía en una abolición del programa de rentas mínimas dirigido a las personas en situación de pobreza y en el establecimiento de una renta garantizada con suplementos económicos que tenía cierta similitud con el impuesto negativo sobre la renta. Sin embargo, y aunque el plan pasase la Cámara de los Representantes de Estados Unidos, fue rechazada en la comisión correspondiente en el Senado. El resultado de estos años fue un debate corto pero intenso que seguiría su cauce por la vía académica sobre la base de cinco experimentos con sistemas de impuestos negativos sobre la renta (cuatro en los Estados Unidos y uno en Canadá).

Mención especial merece el Estado de Alaska. En Alaska, cada persona que cumpla los requisitos de residencia, recibe desde 1982 una parte proporcional de los rendimientos adquiridos a través del Fondo Permanente de Alaska (Alaska Permanent Fund), una cartera de inversiones pública financiada a raíz de los ingresos petroleros del estado. Asimismo, Alaska es el único ejemplo a escala mundial en transformar un recurso natural agotable en un fondo de riqueza soberana que distribuye de manera individual e incondicional los ingresos a todas las personas residentes (Casassas y De Wispelaere, 2012).

En los años 80 fueron surgiendo debates independientes en diversos países europeos que gradualmente entraron en contacto entre sí a través de la creación en el año 1986 de la Basic Income European Network (BIEN), quien publicaba un boletín periódico y organizaba conferencias bianuales. Posteriormente, la creación de diversas asociaciones sobre renta básica a lo largo y ancho del mundo, motivó que en el año 2004, en el congreso realizado en Barcelona, fueran modificadas las siglas de la BIEN y ésta se renombrara como Basic Income Earth Network.

\section{c) La globalización e incorporación de la renta básica incondicional en la agenda política: siglo XXI}

No es casual que el cambio de la red europea a la red mundial se diera durante el siglo Xxi. Es en las últimas dos décadas cuando los debates y estudios sobre la renta básica incondicional han proliferado tanto en ámbitos académicos como en ámbitos políticos, con una aceleración muy notable en los dos últimos años 2017 y 2018. Uno de los motivos por los que la renta básica incondicional ha conseguido un cada vez mayor espacio en la agenda política es, en parte, gracias a los diversos proyectos piloto que se están llevando a cabo en geografías y economías tan dispares como Kenia, Namibia, India, Canadá, Finlandia, Países Bajos, Francia, Escocia o España. Proyectos piloto que, a día de hoy, algunos ya han acabado (debido al límite temporal o a su cancelación), otros aún están en marcha y otros están en proceso de empezar (Bollain, 2018; Reed y Lansley, 2016). 
Los proyectos piloto han servido, como apuntaba, a que la renta básica incondicional se posicionase en la agenda política de casi todas las economías ricas, así como de las menos ricas o pobres. Aún así, no podemos llamarnos a engaño y hay que aclarar que los proyectos piloto cuentan, como mínimo, con 5 grandes limitaciones comunes que no nos permiten apreciar los efectos que una renta básica incondicional pudiera tener sobre la totalidad de la ciudadanía.

Los proyectos piloto son limitados en el tiempo, por lo que no nos permiten observar cómo se comportarían las personas si tuvieran su existencia material garantizada a lo largo de toda su vida. Además, para llevarlos a cabo se utiliza una muestra, generalmente no significativa ni generalizable al total de la población ya que suele ser sesgada (por ejemplo, personas desempleadas de larga duración) y suele estar saturada. En esta muestra sesgada que se escoja tampoco se incluye a personas contribuyentes netas al sistema ya que los proyectos piloto tienen efectos impositivos nulos. Es decir, éstos se financian a través de ayudas, partidas presupuestarias o mediante donaciones. Finalmente, y debido a la limitación temporal, en los proyectos piloto no es observable el efecto que una renta básica incondicional pudiera tener sobre el mercado laboral.

Por el contrario, los proyectos piloto pueden ofrecernos ciertos datos interesantes sobre la posible reducción del nivel de estrés de las personas al tener momentáneamente un ingreso regular garantizado o el posible aumento en el nivel de búsqueda activa de empleo de las personas que toman parte en los experimentos (Bollain, 2018).

Últimamente se puede leer a diversas organizaciones económicas mundiales como el Fondo Monetario Internacional (2017) o el Banco Mundial (2019), a multitud de académicos y académicas o a personas del ámbito político sobre la necesidad de explorar vías alternativas a las rentas mínimas.

En ocasiones, dichos debates en torno a cómo actualizar los diversos Estados de Bienestar se basan en la imagen de un futuro próximo ( 25 años) en el que existe la posibilidad de que hasta un $47 \%$ de los empleos mundiales sean computerizados (Frey y Osborne, 2017). Todo parece indicar que se presenta un futuro donde el desempleo ya no solo estará ligado a la producción o al ciclo económico, sino que cada vez tendrá mayor peso la cantidad de empleos computerizados y, por ende, la variable sobre la distribución del propio empleo resultante. Y ante esta realidad donde la automatización, la digitalización y la robotización se presentan inminentes, a menudo se plantea un futuro donde la existencia material de la ciudadanía no solo esté garantizada a través del empleo o condicionada a políticas activas de inclusión laboral (workfare). Y es en este nuevo marco cuando personas estudiosas en la materia han planteado la renta básica incondicional como pilar de un sistema de protección social alternativo a los actuales (véase, por ejemplo, Butaru, 2017). 
Sin embargo, además de la potencialidad que la renta básica incondicional posee ante un futuro donde, presumiblemente, se dará una destrucción de empleo neto, resulta interesante analizar cómo ésta permite franquear con gran habilidad parte de los problemas de las rentas mínimas identificados anteriormente.

\section{Comparación renta básica incondicional y rentas mínimas}

En este apartado trataremos de analizar el comportamiento de la renta básica incondicional ante las cinco razones principales que provocan el mal funcionamiento de las rentas mínimas (ver apartado 4).

En primer lugar, la renta básica incondicional no debería presentar problemas presupuestarios siempre y cuando el modelo esté diseñado de forma competente. Pese a que la financiación de la renta básica incondicional supone una gran dedicación de recursos, hay que ser conscientes de que no se deben equiparar las transferencias y los gastos. Las transferencias que se realizan son reasignaciones de poder adquisitivo desde la minoría más rica hacia la mayoría social no estrictamente rica. Es decir, el coste neto de financiar una renta básica incondicional es indudablemente menor que el coste bruto. Por eso, en la mayoría de los estudios realizados (Quass y Thieme, 2013; Arcarons et al., 2017; Martinelli, 2017), la renta básica incondicional se financia a raíz de una reforma fiscal. Estudios que demuestran en distintas áreas geográficas cómo un programa de financiación de una renta básica incondicional bien administrado es asequible, factible y altamente consistente.

En segundo lugar, hay que tener en cuenta que la renta básica incondicional abarcaría al conjunto de la ciudadanía y, como afirmó Atkinson (1996), a diferencia de la gran tasa de no-aceptación de los subsidios condicionados, se estima que la misma sea cercana al $0 \%$ cuando se trata de subsidios incondicionales.

En este sentido, es de suponer que el principio de incondicionalidad de la renta básica incondicional le otorga una ventaja frente al déficit de cobertura asociado a las rentas mínimas.

En tercer lugar y en línea con lo sostenido por Van Parijs (2006), no hay nada de humillante y que pueda causar un estigma social en el hecho de otorgar una renta básica incondicional a toda la población como derecho de ciudadanía. Ninguna persona se sentiría "señalada» al recibir una renta básica incondicional ya que su universalidad, frente a la focalización de los subsidios condicionados, permite que la ciudadanía no sea dividida entre aquellas personas que dan y aquéllas que reciben. A diferencia de los exhaustivos controles existentes para acceder a los programas de rentas mínimas actuales, debido a la universalidad y 
a la incondicionalidad de la renta básica incondicional, ninguna persona tendría la necesidad de «desnudarse» ante la administración pública para que determine si es elegible para recibir alguna prestación.

En definitiva, las características de una renta básica incondicional permitirían superar prácticamente por completo los problemas de estereotipación y estigmatización tan característicos de los programas de rentas mínimas, a la vez que ayudarían a fortalecer la solidaridad social reforzando el sentimiento de pertenencia a la comunidad y la cohesión social (Standing, 2008).

Cuando hacíamos referencia a las limitaciones presupuestarias afirmábamos que las transferencias de la renta básica incondicional no son estrictamente gastos sino una reasignación del poder adquisitivo. Sin embargo, en ningún momento podemos suponer que estas transferencias no tengan coste alguno.

La existencia de altos costes de administración en los programas de rentas mínimas en comparación con el presupuesto total asignado, es una parte crucial para que diversos autores (Van Parijs, 2006; Raventós, 2007; Offe, 2005) afirmen que una renta básica incondicional es más eficiente económicamente que los programas de rentas mínimas. Parece sensato pensar que una renta básica incondicional ahorraría costes administrativos ya que simplificaría enormemente la complejidad de los programas de rentas mínimas haciéndolos más transparentes y reduciendo el grado de control y seguimiento.

Van Parijs (2006) afirma que bajo un programa de renta básica incondicional habría costes de administración pero, indudablemente, éstos serían menores a los existentes bajo los programas de rentas mínimas asumiendo la existencia de un sistema computarizado y eficiente en la recaudación de impuestos y en las transferencias de los pagos. Proporcionalmente no hay duda de que los costes en los que una renta básica incondicional incurriría son mucho menores a los de un programa de rentas mínimas ya que el número de personas que acceden al derecho es muy superior. Pero en términos absolutos parece prudente sostener que los costes administrativos de llevar a cabo un programa de renta básica incondicional también son inferiores respecto a uno de rentas mínimas. Garantizar el pago (presumiblemente mensual) de una cuantía a toda la ciudadanía sin tener que realizar ninguna otra comprobación (nivel de renta, seguimiento de búsqueda activa de empleo, condiciones familiares...) no debería suponer gran gasto de personal (Raventós, 2007).

Finalmente, la renta básica incondicional es una herramienta que sortea a la perfección la trampa de la pobreza tan característica de las rentas mínimas. La renta básica incondicional se entiende como un «suelo» o "base» que no es incompatible con otras fuentes de renta como lo son, generalmente, las rentas mínimas. Precisamente por la posibilidad de acumular distintas rentas aparte de la prestación y siempre y cuando dichas fuentes de renta no sean objeto de una im- 
posición fiscal que pueda alcanzar un tipo marginal del $100 \%$, se materializa en que toda persona que trabaje de manera remunerada recibirá más ingresos que una persona que solo perciba la renta básica incondicional.

Por ende, mientras los programas de rentas mínimas son criticados por su poder de disuasión para trabajar de forma remunerada (especialmente para los trabajadores poco cualificados) (Standing, 2008), la renta básica incondicional, gracias a su acumulabilidad, podría ser en muchos casos un estímulo para la búsqueda y aceptación de trabajos remunerados. Y, sin duda, de forma mucho más libre por la parte más débil del contrato laboral.

\section{Conclusiones}

En primer lugar hemos analizado la eficacia de las rentas mínimas y hemos realizado un informe integral detallado de las limitaciones más frecuentes atribuidas a las mismas (restricciones presupuestarias, errores de cobertura, estigmatización de las personas beneficiarias, costes de administración y trampa de la pobreza). De esta forma se ha evidenciado la ineficiencia económica de las rentas mínimas y se ha corroborado que éstas proporcionan una red de seguridad incompleta.

Por otro lado, hemos comparado el comportamiento que la renta básica incondicional tiene ante las limitaciones de las rentas mínimas anteriormente mencionadas. Analizándolas una a una se ha podido demostrar cómo la renta básica incondicional las sortea con gran habilidad gracias a los principios de universalidad, incondicionalidad y acumulabilidad de rentas.

A lo largo del artículo, además, hemos podido constatar cómo existe una gran diferencia de concepción entre las rentas mínimas (o subsidios condicionados, en general) y la renta básica incondicional. Esta diferencia de concepción entre ambas medidas se expresa en términos de libertad. Las rentas mínimas ayudan a las personas una vez han "fracasado». Éstas ofrecen una ayuda ex post a personas que se encuentran en una situación de necesidad a cambio de una contraprestación por las prestaciones percibidas (habitualmente, a través de un proceso de intervención laboral). La mera asistencia ex post conduce irremediablemente a la pérdida de libertad efectiva para quien vive de un salario, y donde se obliga a aceptar el statu quo o a doblegarse ante formas especialmente lesivas para sus intereses de configuración política de los mercados (y más en concreto, del mercado de trabajo). Es decir, la renta básica incondicional supondría también una determinada configuración política de los mercados en beneficio de la parte asalariada del contrato laboral.

La renta básica incondicional, por contra, al ser una asignación monetaria que recibiría toda la población como derecho de ciudadanía y de residen- 
cia acreditada, supone garantizar la existencia material de la ciudadanía ex ante. Con la renta básica se consigue la lógica incondicional de las medidas que actúan ex ante, no obligando a muchas personas a comportarse como «sumisas suplicantes». De manera que se garantiza a toda la población la existencia material de entrada, "por el mero hecho de ser moradores de un mundo cuya riqueza ha sido producida socialmente y conviene repartir sin exclusiones» (Casassas y Raventós, 2018). El carácter incondicional de la renta básica supone otorgar un poder de negociación que implica aumentar la libertad efectiva para la inmensa mayoría de la población no estrictamente rica.

\section{Bibliografía}

Arcarons, J., Raventós, D. y Torrens, L. (2017). Renta básica incondicional. Una propuesta de financiación racional y justa. Barcelona: Ediciones del Serbal.

Arcarons, J., Raventós, D. y Torrens, L. (2018). «La Renta Básica, ¿¿da igual?», Sin Permiso. Disponible en: http://www.sinpermiso.info/textos/la-renta-basica-da-igual. Consultado por última vez: 25/10/2018.

Atkinson, A.B. (1996). "The case for a participation income», The Political Quarterly, 67(1), 67-70.

Aust, A. y Arriba, A. (2005). "Towards Activation? Social Assistance Reforms and Discourses», en Taylor-Gooby P. (ed.), Ideas and Welfare State Reform in Western Europe. Nueva York: Palgrave Macmillan.

Bahle, T., Pfeifer, M. y Wendt, C. (2010). "Social assistance», en Castles, F. G., Leibfried, S., Lewis, J., Obinger, H., y Pierson, C. (eds.), The Oxford handbook of the welfare state. Oxford: Oxford University Press.

Banco Mundial (2019). World Development Report 2019: The Changing Nature of Work. Washington, DC: The World Bank.

Bargain, O., Immervoll, H. y Viitamäki, H. (2012). «No claim, no pain. Measuring the non-take-up of social assistance using register data», The Journal of Economic Inequality, 10(3), 375-395.

BeNFIELD, W. (2007). Indicator targeting: The jamaica food stamp programme. Artículo presentado en SALISES $8^{\text {th }}$ Annual Conference, The University of the West Indies. Trinidad y Tobago.

Bird, E. J., Kayser H. y Frick J. R. (1999). The Immigrant Welfare Effect, Take-up or Eligibility? Bonn: IZA.

Blanchard, O. y Wolfers, J. (2000). «Shocks and Institutions in the Rise of European Unemployment: The Aggregate Evidence», Economic Journal, 110(1), 1-33.

Bollain, J. (2017). «La Renta Básica y la vagancia de ciertos mitos», Sin Permiso. Disponible en: http://www.sinpermiso.info/textos/la-renta-basica-y-la-vagancia-de-ciertosmitos. Consultado por última vez: 04/10/2018.

Bollain, J. (2018). «El estrepitoso fracaso del proyecto finlandés de Renta Básica. ¿Cómo manipular la realidad?», Sin Permiso. Disponible en: http://www.sinpermiso.info/ textos/el-estrepitoso-fracaso-del-proyecto-finlandes-de-renta-basica-como-manipularla-realidad. Consultado por última vez: 24/09/2018 
Bramley, G., Lancaster, S. y Gordon, D. (2000). «Benefit take-up and the geography of poverty in Scotland», Regional Studies, 34(6), 507-519.

Butaru, L. T. (2017). "Towards a universal basic income», An evolutionary approach. Studia Universitatis Babes-Bolyai Europaea, 62(3).

Casassas, D. y De Wispelaere, J. (2012). "The Alaska Model: A Republican Perspective», en Widerquist, K. y Howard, M. W. (eds.), Alaska's Permanent Fund Dividend. Exploring the Basic Income Guarantee. Nueva York: Palgrave Macmillan.

Casassas, D. y Raventós, D. (2018). «La viabilidad de la renta básica en el Reino de España», en Standing, G. (epílogo), La renta básica. Un derecho para todos y para siempre. Barcelona: Pasado y Presente.

Charlier, J. (1848). Solution du problème social ou constitution humanitaire, basée sur la loi naturelle, et précédée de l'exposé des motifs. Bruselas: Greuse.

Cohen, W. J. y Friedman, M. (1972). Social Security: Universal or Selective. Washington, DC: American Enterprise Institute.

Cook, F. y BARrett, E. (1992). «Beliefs about recipient deservingness and program effectiveness as explanations of support», en Cook, F. y Barrett, E. (eds.), Support for the American welfare state: Views of congress and the public. Nueva York: Columbia University Press.

Crouch, C. (1999). Social change in western Europe. Oxford: Oxford University Press.

Currie, J. (2004). The Take Up of Social Benefits. Cambridge, MA: National Bureau of Economic Research.

Devereux, S., Masset, E., Sabates-Wheeler, R., Samson, M., Rivas, A. M., y Te LinTELO, D. (2017). "The targeting effectiveness of social transfers», Journal of Development Effectiveness, 9(2), 162-211.

Eurostat (1994). Poverty Statistics in the Late 1980s. Luxemburgo: Statistical Office of the European Communities.

Euzeby, C. (1991), Le revenu minimum garanti. Paris: La Découverte.

Fondo Monetario Internacional (2017). Fiscal Monitor. Tackling Inequality. Washington, DC: International Monetary Fund.

Frazer, H. y Marlier, E. (2016). Minimum Income Schemes in Europe. A study of national policies, European Social Policy Network (ESPN). Bruxelles: Commission européenne.

Frey, C. B. y Osborne, M. A. (2017). "The future of employment: how susceptible are jobs to computerisation?», Technological forecasting and social change, 114, 254-280.

GiLens, M. (1999) Why Americans hate welfare: Race, media, and the politics of antipoverty policy. Chicago: University of Chicago Press.

Grosh, M. (1994). Administering Targeted Social Programmes in Latin America: From Platitudes to Practice. Washington, D.C: World Bank.

Gustafsson, B. (2002). "Assessing non-use of social assistance», European Journal of Social Work, 5(2), 149-158.

Hernanz, V., Malmerbet, F. y Pellizzari, M. (2004). «Take-Up of Welfare Benefi ts in OECD Countries: A Review of Evidence», Social, Employment, and Migration Working Paper 17. Paris: Organisation for Economic Co-operation and Development.

ImMervoll, H. (2010), "Minimum-Income Benefits in OECD countries: policy design, effectiveness and challenges», OECD Social Employment and Migration Working Papers, 100.

Jacobs, L. R. y Shapiro, R. Y. (1994). "Questioning the conventional wisdom on public opinion toward health reform", Political Science \& Politics, 27(2), 208-214. 
Korpi, W. y Palme, J. (1998). «The paradox of redistribution and strategies of equality: Welfare state institutions, inequality, and poverty in the Western countries», American sociological review, 63, 661-687.

LEY I 8/2008, de 23 de diciembre, "para la Garantía de Ingresos y para la Inclusión Social». Boletín Oficial del País Vasco N.o 250/2008 del 31 de diciembre de 2008.

Lødemel, I. y TRICKey, H. (2001). 'An offer you can't refuse': workfare in international perspective. Bristol: The Policy Press.

LoI $\mathrm{N}^{\circ}$ 88-I088, de 1 diciembre 1988 «relative au revenu minimum d'insertion». Journal officiel de la République française del 3 de diciembre de 1988.

Martineldi, L. (2017). «The fiscal and distributional implications of alternative universal basic income schemes in the UK», Institute for Policy Research Working Paper. Bath: University of Bath.

Matsaganis, M., Levy, H., y Flevotomou, M. (2010). «Non-take up of social benefits in Greece and Spain», Social Policy \& Administration, 44(7), 827-844.

Milner, E. M. y Milner, D. (1918). Scheme for a State Bonus: A Rational Method of Solving the Social Problem. Darlington: North of England Newspaper Company.

Ministerio de Hacienda (2018). Presupuestos Generales del Estado, Gobierno de España. Disponible en: http://www.hacienda.gob.es/es-ES/Areas\%20Tematicas/Presupuestos\%20Generales\%20del\%20Estado/paginas/Presupuestos.aspx. Consultado por última vez: 3/10/2018.

Moro, T. (1516). Utopia. Lovaina

Nelson, K. (2007). «Minimum Income Protection and European Integration: Trends and Levels of Minimum Benefits in Comparative Perspective 1990-2005", International Journal of Health Services, 38(1), 103-124.

Nelson, K. (2010). «Social assistance and minimum income benefits in old and new EU democracies", International Journal of Social Welfare, 19(4), 367-378.

Neumann, U. y Hertz, M. (1998). Verdeckte Armut in Deutschland. Frankfurt am Main: Institut für Sozialberichterstattung und Lebenslagenforschung.

Offe, C. (2005) «Wasteful welfare transactions: why basic income security is fundamental», en Standing, G. (ed.), Promoting income security as a right: Europe and North America. Londres: Anthem.

Orús, A. (productor y director) (2018). RBUI, nuestro derecho a vivir [documental]. España: Alvaro Orús.

Paine, T. (1797) «Agrarian Justice», en Cunliffe J. y Erreygers G. (eds.) (2004), The Origins of Universal Grants. Londres: Palgrave Macmillan.

QuaAs, G., y Thieme, S. (2013). «Das bedingungslose Grundeinkommen-eine ökonometrische Analyse seiner volkswirtschaftlichen Wirkungen», Wirtschaftsdienst, 93, 598-605.

RAINWATER, L. (1982). «Stigma in income-tested programs», en Garfinkle, I. (ed.), Income-tested programs: The case for and against. Nueva York: Academic Press.

Raventós, D. (2000). «El salario de toda la ciudadanía», Claves de razón práctica, 106, $1-11$.

Raventós, D. (2006). «Renta Básica de ciudadanía. Filosofía, economía y política», Papeles del Este, 12, 1-25.

Raventós, D. (2007). Las condiciones materiales de la libertad. Barcelona: El Viejo Topo.

Real Decreto Legislativo i/i994, de 20 de junio, "por el que se aprueba el Texto Refundido de la Ley General de la Seguridad Social». Boletín Oficial del Estado del 29 de junio. 
Red Renta Básica (20i8). ¿QUÉ es la RB? Disponible en: http://www.redrenta BASICA.ORG/RB/QUE-ES-LA-RB/. CONSULTADO POR ÚLTIMA VEZ: O8/IO/2OI 8 .

Reed, H. y Lansley, S. (2016). Universal Basic Income: An idea whose time has come? Londres: Compass.

Riphahn, R. T. (2001). "Rational poverty or poor rationality? The take-up of social assistance benefits", Review of income and wealth, 47(3), 379-398.

Sainsbury, D. y Morissens, A. (2002). "Poverty in Europe in the mid-1990s: the effectiveness of means-tested benefits", Journal of European Social Policy, 12(4), 307-327.

Son, H (2008). Conditional Cash Transfer Programs: An Effective Tool for Poverty Alleviation? Mandaluyong, Filipinas: Asian Development Bank.

Standing, G. (2003). Minimum income schemes in Europe. Ginebra: International Labour Organization.

Standing, G. (2008). «How cash transfers promote the case for basic income», Basic Income Studies, 3(1), 5.

Stapleton, D. C., O’day, B. L., Livermore, G. A. y Imparato, A. J. (2006). «Dismantling the poverty trap: Disability policy for the twenty-first century», The Milbank Quarterly, 84(4), 701-732.

Stuber, J., y Kronebusch, K. (2004). «Stigma and other determinants of participation in TANF and Medicaid», Journal of Policy Analysis and Management, 23(3), 509-530.

Terracol, A. (2002). Analyzing the take-up of means-tested benefits in France. París: Université de Paris.

Therborn, G. (1995). European modernity and beyond: the trajectory of European societies, 1945-2000. Londres: Sage.

Van der Berg, S., Siebrits, F. y Lekezwa, B. (2010). "Efficiency and equity effects of social grants in South Africa", Stellenbosch Economic Working Papers 15/10. Stellenbosch: Stellenbosch University.

VAN OORschot, W. (1991). "Non-take-up of social security benefits in Europe», Journal of European social policy, 1(1), 15-30.

VAN OORschOT, W. (1995). Realizing Rights: a multilevel approach to the non-take-up of meanstested benefits. Aldershot: Ashgate Publishing.

VAN OORschot, W. (1998). "Failing selectivity: On the extent and causes of non-take-up of social security benefits», en Andreß, H. J. (ed.), Empirical poverty research in a comparative perspective. Aldershot: Ashgate Publishing.

Van Parijs, P. (2006). "Basic Income: A simple and powerful idea for the 21st century», en Wright E. O. (ed.), Redesigning Distribution. Basic Income and Stakeholder Grants as Cornerstones for an Egalitarian Capitalism. Londres: Verso.

VAn Parijs, P. y Vanderborght, Y. (2017). Basic income: A radical proposal for a free society and a sane economy. Cambridge, MA: Harvard University Press.

Weiner, B., Perry, R. P., y Magnusson, J. (1988). "An attributional analysis of reactions to stigmas", Journal of personality and social psychology, 55(5), 738. 